\title{
In-Situ Transmission Electron Microscopy Investigation on the Vapor-Solid Growth of $\mathrm{ZnO}$ Nanowires
}

\author{
Xing $\mathrm{Li}^{1 *}$, Shaobo Cheng ${ }^{2}$, Jing $\mathrm{Zhu}^{3}$ and Qing Chen ${ }^{4}$ \\ 1. Key Laboratory of Material Physics, Ministry of Education, Department of Physics and Engineering, \\ Zhengzhou University, Zhengzhou, P. R. China \\ 2. Department of Condensed Matter Physics and Materials Science, Brookhaven National Laboratory, \\ Upton, NY, USA \\ 3. School of Materials Science and Engineering, Tsinghua University, Beijing, P. R. China \\ 4. Key Laboratory for the Physics and Chemistry of Nanodevices and Department of Electronics, Peking \\ University, Beijing, P. R. China \\ * Corresponding author: xingli@zzu.edu.cn
}

Due to its unique semiconducting, piezoelectric, biocompatible and optoelectronic properties, $\mathrm{ZnO}$ nanomaterials, especially wurtzite (WZ) nanowires (NWs) with $<0001>$ growth direction, are promising candidates for a wide range of applications in ultraviolet optoelectronics [1], electromechanics [2,3], optoelectronics and gas sensors [4]. ZnO NWs and a variety of more complex morphologies have been fabricated using the vapor-solid (VS) method in the absence of a catalyst. During the growth of $\mathrm{ZnO}$ NWs, especially in the VS transport process, the atoms or molecules contributing to the growth can either directly attach to the tip or diffuse along the side walls of the nanowires and the diffusion process is very important for the morphology of the grown NWs. Moreover, coalescence of nanocrystals is an important phenomenon in the early synthesis stage of nanomaterials. While VS methods are important for the synthesis of catalyst-free NWs, direct observation of $\mathrm{ZnO} \mathrm{NW}$ growth process and the dynamic evolution of the coalescence process of $\mathrm{ZnO}$ nanocrystals at atomic scale still lack experimental demonstration.

Here, high resolution transmission electron microscope (HRTEM) equipped with spherical aberration correction is used to investigate the solid-source growth, surface stability and coalescence of $\mathrm{ZnO}$ nanostructures under electron beam irradiation. The $\mathrm{ZnO}$ film grown by the pulsed laser deposition method is acting as the source material for the NW growth and the stacking fault in the $\mathrm{ZnO}$ film is indicated as a reference for the quantitative analysis on the growth process. With the increase of the irradiation time, the adatoms produced by e-beam induced sputtering move along the (10 $\overline{1} 0$ ) surface, resulting in a layer-by-layer growth of (0002) surface. The growth rate of the $\mathrm{ZnO} \mathrm{NW}$ is estimated to be $0.26 \mathrm{~nm} / \mathrm{s}$. With direct in situ HRTEM observations, we find that during the solid-source growth process, the atoms remaining absorbed and/or being reabsorbed from the vicinity vacuum can reach the top (0001) surface via a diffusion process along the sidewalls of the NW. Due to the larger cleavage energy of the polar surfaces [5], an anisotropic growth only along the [0001] direction is observed. Driven by the reduction of surface and interfacial energy, the coalescence of two $\mathrm{ZnO}$ nanocrystals is realized by in plane rotations. These results provide detailed information about the atomic translational and rotational movement of $\mathrm{ZnO}$ in atomic resolution, while the layer-by-layer growth induced by surface atom diffusion may contribute to the understanding of the role of the diffusion process in the $\mathrm{ZnO} \mathrm{NW}$ growth. 


\section{References:}

[1] M. H. Huang, et al., Science 292 (2001), p.1897.

[2] X. Li, et al., Adv. Mater. Interfaces 6 (2018), 1701246.

[3] X. Li, et al., Nanomaterials 8 (2018), 188.

[4] Q. Wan, et al., Appl. Phys. Lett. 84 (2004), 3654.

[5] B. Meyer and D. Marx, Phys. Rev. B 67 (2003), 035403.

[6] The authors acknowledge funding from National Science Foundation of China (No.11804304) and China Postdoctoral Science Foundation (2017M622371).

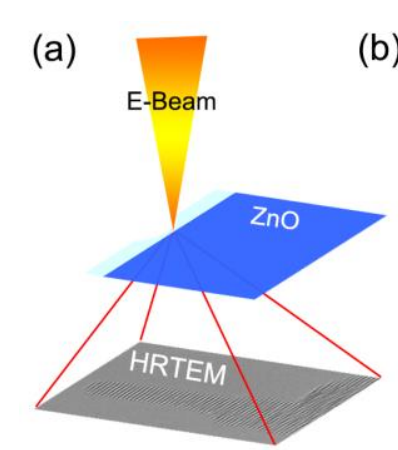

(d)

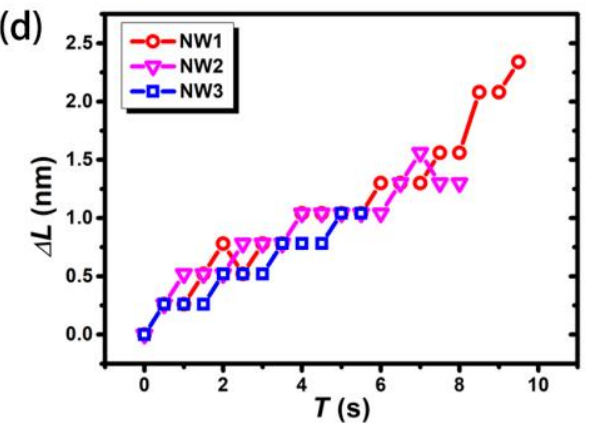

(b)

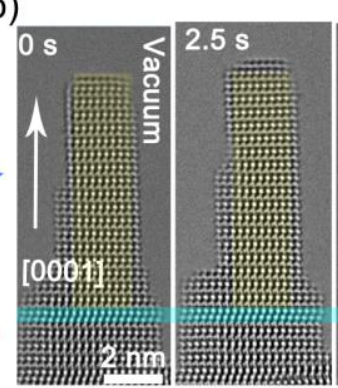

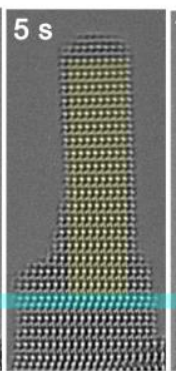
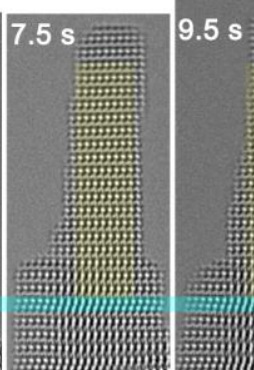

(e)

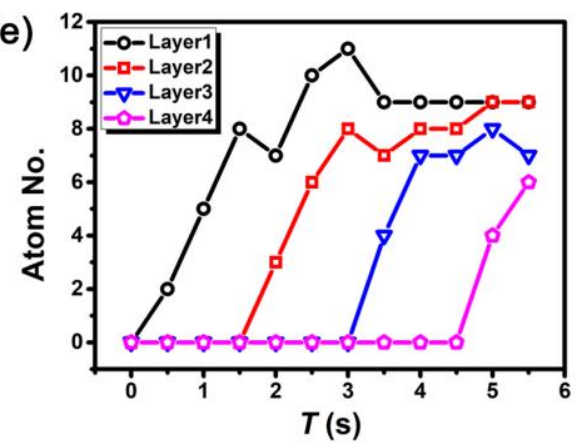

Figure 1. Layer-by-layer growth of ZnO NWs. (a) Experimental setup for the in situ HRTEM study. (b) Layer-by-layer growth of a $\mathrm{ZnO} \mathrm{NW}$ under e-beam irradiation; a stacking fault is marked with blue color (c) Schematic illustration of the e-beam induced sputtering and migration of $\mathrm{Zn}-\mathrm{O}$ atoms. (d) The relationship between the length change $(\Delta L)$ and the e-beam irradiation time $(T)$ of three different NWs. (e) The change of $\mathrm{Zn}$ atom numbers on each growing layer with irradiation time $(T)$.
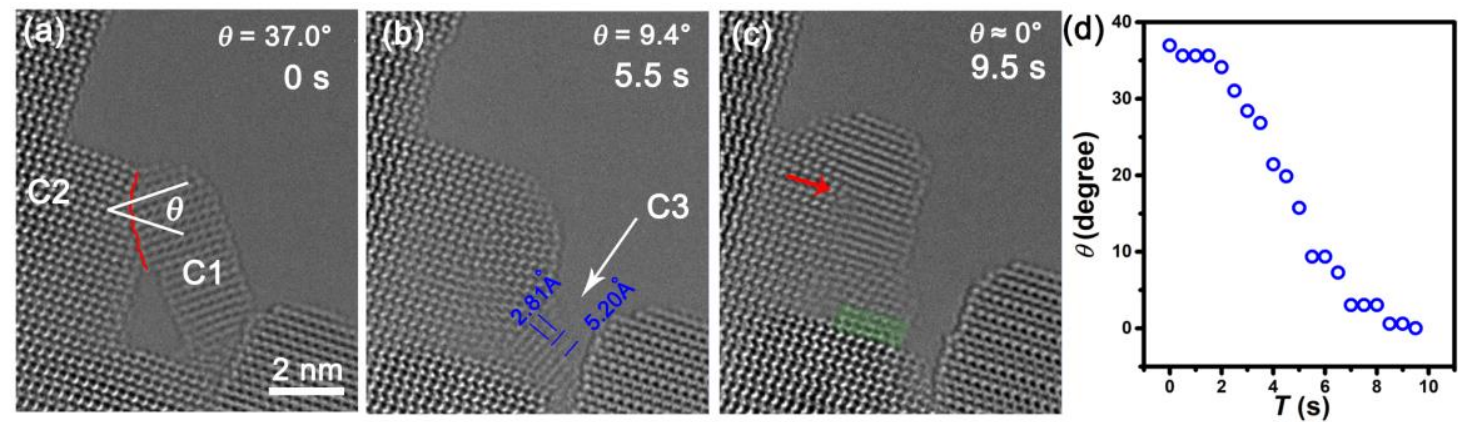

Figure 2. Coalescence of two $\mathrm{ZnO}$ crystals. (a) HRTEM image showing the angle $\theta$ between the (0002) planes of Crystal 1 (C1) and Crystal 2 (C2). (b) The rotation of $\mathrm{C} 1$ resulting in the decrease of $\theta$. The newly formed Crystal 3 (C3) are indicated by the arrow. (c) The rotation of C3. (d) The relationship between $\theta$ and the irradiation time $T$. 